Ref. SISSA 45/2003/FM

\title{
Gaudin Models and Bending Flows: a Geometrical Point of View
}

\author{
Gregorio Falqui and Fabio Musso \\ SISSA, Via Beirut 2/4, I-34014 Trieste, Italy \\ falqui@sissa.it, musso@sissa.it \\ November 20, 2018
}

\begin{abstract}
In this paper we discuss the bihamiltonian formulation of the (rational XXX) Gaudin models of spin-spin interaction, generalized to the case of $\operatorname{sl}(r)$-valued "spins". In particular, we focus on the homogeneous models. We find a pencil of Poisson brackets that recursively define a complete set of integrals of the motion, alternative to the set of integrals associated with the "standard" Lax representation of the Gaudin model. These integrals, in the case of $s u(2)$, coincide wih the Hamiltonians of the "bending flows" in the moduli space of polygons in Euclidean space introduced by Kapovich and Millson. We finally address the problem of separability of these flows and explicitly find separation coordinates and separation relations for the $r=2$ case.
\end{abstract}

AMS Classification numbers: 70H06, 37K10, 70H20.

\section{Introduction}

In [12, M. Gaudin proved the integrability of $N$-site $s u(2)$ (quantum) spin Hamiltonians of the form

$$
\mathcal{H}=\sum_{j<l=1}^{N} \frac{c_{j}-c_{l}}{a_{j}-a_{l}} \vec{\sigma}_{j} \cdot \vec{\sigma}_{l},
$$

where $\vec{\sigma}=\left[\sigma_{x}, \sigma_{y}, \sigma_{z}\right]$ are Pauli matrices. This property follows from the fact that one can write the Gaudin Hamiltonian $\mathcal{H}$ as

$$
\mathcal{H}=\sum_{j=1}^{N} c_{j} H_{j} \quad \text { with } \quad H_{j}=\sum_{l \neq j} \frac{\vec{\sigma}_{j} \cdot \vec{\sigma}_{l}}{a_{j}-a_{l}}
$$


and check that the $H_{j}$ 's define a set of $N-1$ commuting observables. Since fixing the values of the $N$ Casimirs $C_{j}=\left|\vec{\sigma}_{i}\right|^{2}$ the system has $N$ degrees of freedom, the $N-1$ quantities $H_{j}$, together with, e.g., $S_{z}=\sum_{i=1}^{N} \sigma_{i}^{z}$, provide a complete set of mutually commuting observables.

A relevant member of this class of Hamiltonians is obtained when one chooses $c_{k}$ to be proportional to $a_{k}$ for all $k$ 's, so that, up to a rescaling, the Hamiltonian (1.1) becomes

$$
\mathcal{H}=\sum_{j, l=1}^{N} \vec{\sigma}_{j} \cdot \vec{\sigma}_{l} .
$$

This is the Hamiltonian of the XXX rational homogeneous Gaudin model.

This system is not only integrable, but maximally superintegrable. One can understand this stronger property as follows (see, e.g [15]): since the "physical" Hamiltonian (1.3) is independent of the parameters, the choice of the $a_{k}$ 's in the definition of the commuting integrals $H_{j}$ is arbitrary (provided $a_{i} \neq a_{j}, i \neq j$ ). So, choosing another set of parameters $b_{k} \neq a_{k}$ and considering $\tilde{H}_{l}=\sum_{l \neq j} \frac{\vec{\sigma}_{j} \cdot \vec{\sigma}_{l}}{b_{j}-b_{l}}$ one can define the two sets of complete commuting quantities:

$$
\left\{\mathcal{H}, H_{1}, \ldots, H_{N-2}, S_{z}\right\} ; \quad\left\{\mathcal{H}, \tilde{H}_{1}, \ldots, \tilde{H}_{N-2}, S_{x}\right\}
$$

Since for generic choices of the sets $a_{k}, b_{k}$ the observables

$$
\left\{\mathcal{H}, H_{1}, \ldots, H_{N-2}, \tilde{H}_{1}, \ldots, \tilde{H}_{N-2}, S_{z}, S_{x}\right\}
$$

are algebraically independent, the model is indeed maximally superintegrable.

Recently it was pointed out independently by various authors [4, 18, that with (1.3) it is possible to associate a set of commuting integrals independent of the parameters. Such operators are of the form:

$$
I_{k-1}=\sum_{j, l=1}^{k} \vec{\sigma}_{j} \cdot \vec{\sigma}_{l} \quad k=2, \ldots, N
$$

and, together with, $S_{z}$ they form a complete set of involutive integrals for $\mathcal{H}$.

In a completely different context the classical version of the integrals (1.4), namely

$$
K_{j}=\frac{1}{2} \operatorname{Tr}\left(\left(\sum_{i=1}^{j+1} A_{i}\right)^{2}\right),
$$

where $A_{1}, \ldots A_{N}$ are generic elements of $s u(2)$, were considered by Kapovich and Millson [17. These authors (see also [11) studied the moduli space of $N+3$ sided polygons in $\mathbb{R}^{3}$, and (implicitly) showed that it coincides with a suitable Marsden-Weinstein quotient (with respect to the diagonal action of $S U(2)$ ) of the phase space of the $N+3$ site $s u(2)$-Gaudin models. They remarked that 
such a space possesses a natural Hamiltonian structure, and integrated, via action-angle variables methods, the flows associated with the integrals $K_{l}, l=$ $1, \ldots, N$. It is worthwhile to remind the intriguing representation of such flows: if one draws, from a chosen vertex, the $N$ possible diagonals of an $N+3$-sided polygon, the flow associated with the Hamiltonian $K_{k}$ geometrically represents the bending of one side of the polygon along the $k$-th diagonal (the other side being kept fixed), whence the name of "bending flows".

The Gaudin system (1.1) admits various generalizations. Gaudin himself pointed out that the integrals (1.2) can be generalized to any semisimple Lie algebra $\mathfrak{g}$. Clearly, if the rank of $\mathfrak{g}$ is greater than 1 , the number of such integrals is not enough to ensure complete integrability. The missing integrals have been shown by Jurčo [16] and Sklyanin [25] to be provided by the spectral invariants of a suitable Lax matrix, whose classical counterpart is

$$
L_{\text {rat }}=\sum_{i=1}^{N} \frac{A_{i}}{\lambda-a_{i}}
$$

where $a_{i} \neq a_{j}, i \neq j$ and the $A_{i}$ are generic elements of $\mathfrak{g}$. In terms of the Lax matrix (1.6) the generalization of the Hamiltonian (1.3) reads:

$$
H_{G}=\sum_{i=1}^{N} r e s_{\left.\right|_{\lambda=a_{i}}} \operatorname{Tr}\left(\lambda L_{\text {rat }}^{2}\right)=\sum_{j \neq i} \operatorname{Tr}\left(A_{i} A_{j}\right) .
$$

Another straightforward generalization of this model is obtained adding a constant term $\sigma$ to the Lax matrix. In the $s u(2)$ case, this is equivalent to adding to the Hamiltonians (1.3) a term describing the interaction of the spins with a magnetic field with a constant direction in each site but with different intensity. In this case one speaks of "inhomogeneous" Gaudin magnet. The complete integrability and separability of these systems, (for the $\mathfrak{g}=\operatorname{sl}(n)$ case) was studied and proved in [26, 13, 24.

The aim of this paper is to frame the analysis of the Gaudin models, as well as of the Hamiltonians (1.5) of the bending flows of Kapovich and Millson, in the scheme of bihamiltonian geometry as advocated by Gel'fand and Zakharevich 14, and to show how one can use this scheme to explicitly integrate the model for $\mathfrak{g}=s l(2)$. We will consider only the classical models, and consider the complexified case (that is, we will study the Gaudin system associated with a complex semisimple Lie algebra $\mathfrak{g}$ ).

Our first task will be to briefly show how, using nowadays standard results of the theory of $r$-matrices on loop algebras (see, e.g., [23]), one can provide the phase space of the (inhomogeneous) Gaudin magnet with a bihamiltonian structure, selecting it out of a multi-parameter family of Poisson structures. This structure gives rise, according to the GZ scheme, to the integrals associated with the Lax matrix of Jurčo and Sklyanin (1.6). 
Then we will construct, in the homogeneous case, another bihamiltonian structure, non compatible (in a sense to be precised later) with the abovementioned family, whose GZ analysis gives rise, in the $s l(2)$ case, to the parameter independent integrals (1.5). Since such additional bihamiltonian structure is still constructed within a Lie-theoretical setting, we will be able to straightforwardly apply this scheme to $\mathfrak{g}=s l(r)$, with arbitrary $r$. In this way, we will be able to find a sufficient number of commuting integrals to be added to the "generalized bending Hamiltonians" $I_{k}$, yielding a complete family of integrals alternative to the "standard" family obtained by Sklyanin and Jurčo.

The GZ analysis of such a model will finally lead us to introduce a kind of Lax matrices for such flows and to show that the Hamilton-Jacobi equations associated with the $s l(2)$ bending Hamiltonians are separable by computing explicitly the separation variables and the separation relations.

\section{GZ analysis of Gaudin models}

The Gel'fand-Zakharevich (GZ) scheme [14 for integrating a bihamiltonian system can be seen as a particularly efficient scheme to implement the LenardMagri recursion for manifolds endowed with a pair of compatible Poisson brackets none of which is symplectic (i.e., non-degenerate).

One considers a manifold $M$ endowed with a pair of compatible Poisson tensors $P_{1}-\lambda P_{0}$, or, in other words, a pencil of Poisson brackets

$$
\{f, g\}_{\lambda}=\{f, g\}_{P_{1}}-\lambda\{f, g\}_{P_{0}}=\left\langle d f,\left(P_{1}-\lambda P_{0}\right) d g\right\rangle .
$$

(where $\langle\cdot, \cdot\rangle$ is the canonical pairing between $T^{*} M$ and $T M$ ), and assumes that the kernel of the generic element of the Poisson pencil be $k$-dimensional. Let $C_{1}, \ldots, C_{k}$ be independent Casimir functions of $P_{0}$. The GZ method, roughly speaking, suggests to use these Casimirs as "starting" elements for Lenard chains yielding (under some technical additional conditions), via the method of bihamiltonian iteration, families of functions $\left\{H_{m}^{(a)}\right\}_{a=1, \ldots, k}^{m}=0, \ldots$, such that for any function $F$ on $M$, and $a=1, \ldots, k$,

$$
\left\{F, H_{m}^{(a)}\right\}_{P_{0}}=\left\{F, H_{m-1}^{(a)}\right\}_{P_{1}}, \quad \text { with } H_{0}^{(a)}=C_{a} .
$$

As a consequence of the bihamiltonian iterative scheme and of the fact that all Lenard chains start with a Casimir function of $P_{0}$ (they are "anchored", in the language of [14), all these functions are mutually in involution with respect to both Poisson brackets. Obviously, the maximal number of independent functions one may hope to get in this way is $N_{\max }=\frac{1}{2}(\operatorname{dim} M+k)$. If this is indeed the case, the geometric scheme herewith outlined defines families of completely integrable systems in the Liouville sense. Indeed, let us suppose that 
the GZ method provides us with $k$ families of mutually commuting independent functions

$$
\left\{H_{m}^{(a)}\right\}_{a=1, \ldots, k}^{m=0, \ldots, n_{a}}, \quad \text { with } \sum_{a=1}^{k} n_{a}=\frac{1}{2}(\operatorname{dim} M+k) .
$$

Let $\mathcal{H}$ be a generic element in the ring generated by such commuting functions, and let $X_{\mathcal{H}}=P_{0} d \mathcal{H}$ be the corresponding Hamiltonian vector field. Let us consider a generic symplectic leaf $S \subset M$ of $P_{0}$; it is a $d_{s}=\operatorname{dim} M-k$ dimensional manifold, with the natural symplectic form induced by the Poisson structure $P_{0}$. $X_{\mathcal{H}}$ clearly restricts to $S$, and, as a consequence of the bihamiltonian iteration on $M$, comes equipped with $\frac{1}{2}(\operatorname{dim} M+k)-k=\frac{1}{2} d_{S}$ integrals in involution, given by the restriction to $S$ of the functions $\left\{H_{m}^{(a)}\right\}_{a=1, \ldots, k}^{m=1, \ldots, n_{a}}$. As a consequence of the genericity assumption on the symplectic leaves, these functions will be independent on $S$ as well and give the complete family of involutive integrals required by the Liouville theorem.

The aim of this Section is to frame the (general, that is, inhomogeneous) Gaudin model within the bihamiltonian scheme, and to reinterpret its complete integrability within the theoretical framework of the GZ analysis briefly sketched above. The manifolds we will consider will be Cartesian products of a Lie algebra $\mathfrak{g}^{N}$, and the Poisson pencils suitable linear pencils of $\mathfrak{g}^{N}$. Since these results are essentially known in the literature, we will mostly limit ourselves to state results and sketch proofs, referring to [23, 22] for a more general setting, and to 10] for the explicit study of the 3-particle $s l(2)$-case.

\subsection{Notations and Conventions}

Let us briefly recall the notion of Lie-Poisson brackets associated with a Lie algebra and fix some notations and conventions we will use throughout the paper.

If $\mathfrak{g}$ is a Lie algebra, its dual $\mathfrak{g}^{*}$ comes equipped with the standard Lie-Poisson structure:

$$
\{F, G\}(A)=\langle A,[d F, d G]\rangle=\langle d F, P d G\rangle, \quad F, G \in C^{\infty}\left(\mathfrak{g}^{*}\right) .
$$

If $\mathfrak{g}$ is semisimple we can identify $\mathfrak{g}^{*}$ with $\mathfrak{g}$. Indeed, we can associate a matrix $X_{A}$ with any element $A \in \mathfrak{g}^{*}$. considering, e.g., the fundamental representation of the algebra $\mathfrak{g}$, and taking the trace form as a bilinear non-degenerate pairing

$$
\left\langle A, X_{B}\right\rangle=\operatorname{Tr}\left(X_{A} \cdot X_{B}\right)
$$

From now on we will implicitly use this identification, and write $A, B \ldots$ instead of $X_{A}, X_{B}, \ldots$ for simplicity of notation. Using the ciclicity of the trace, the Hamiltonian vector field associated by (2.3) with a smooth function $F$ is 
represented by:

$$
P d F=\dot{A}=\left[A, \frac{\partial F}{\partial A}\right],
$$

where the symbol $\frac{\partial F}{\partial A}$ denotes the matrix satisfying, for any $\Xi$ in $\mathfrak{g}$,

$$
F(A+t \Xi)=F(A)+t \cdot \operatorname{Tr}\left(\frac{\partial F}{\partial A} \cdot \Xi\right)+o(t) .
$$

If we take the direct product of $N$ copies of $\mathfrak{g}$, the standard Lie-Poisson structure becomes:

$$
\{F, G\}\left(A_{1}, \ldots, A_{N}\right)=\sum_{i=1}^{N}\left\langle A_{i},\left[\frac{\partial F}{\partial A_{i}}, \frac{\partial G}{\partial A_{i}}\right]\right\rangle
$$

and the Hamiltonian vector field associated with a function $F$ is

$$
\dot{A}_{i}=\left[A_{i}, \frac{\partial F}{\partial A_{i}}\right], \quad i=1, \ldots, N .
$$

We can write the above equation in the form:

$$
\frac{\partial A_{i}}{\partial t}=\left(X_{F}\right)_{i}=(P d F)_{i}=\sum_{j, k} p_{i j k}\left[A_{k}, \frac{\partial F}{\partial A_{j}}\right] \text { with } p_{i j k}=\delta_{i j} \delta_{j k} .
$$

We will also often write $P$ (and other Poisson tensors) representing its action on the differential of a function by means of the matrix symbolic form:

$$
\left(\begin{array}{c}
\dot{A}_{1} \\
\dot{A}_{2} \\
\vdots \\
\dot{A}_{N}
\end{array}\right)=\left(\begin{array}{cccc}
{\left[A_{1}, .\right]} & 0 & \ldots & 0 \\
0 & {\left[A_{2}, .\right]} & \ldots & 0 \\
\vdots & \vdots & & \vdots \\
0 & 0 & \ldots & {\left[A_{N}, .\right]}
\end{array}\right) \cdot\left(\begin{array}{c}
\frac{\partial F}{\partial A_{1}} \\
\frac{\partial F}{\partial A_{2}} \\
\vdots \\
\frac{\partial F}{\partial A_{N}}
\end{array}\right) .
$$

For this reason, we will term the standard Lie-Poisson tensor $P$ on $\mathfrak{g}^{N}$ the diagonal Poisson tensor.

\subsection{A Bihamiltonian Structure of the Gaudin model}

A bihamiltonian structure for rational Gaudin models can be obtained using the following argument. Let us consider the map $\left\{A_{i}\right\} \longrightarrow\left\{B_{i}\right\}$ that sends the rational Lax matrix

$$
L_{r a t}=\sigma+\sum_{i=1}^{N} \frac{A_{i}}{\lambda-a_{i}}
$$


in the polynomial Lax matrix

$$
L_{\text {poly }}=\lambda^{n} \sigma+\sum_{i=0}^{N-1} B_{i} \lambda^{i}=\left(\prod_{i=1}^{N}\left(\lambda-a_{i}\right)\right) \cdot L_{\text {rat }}
$$

given explicitly by

$$
\begin{array}{r}
B_{l}=(-1)^{N-l-1} \sum_{i=1}^{N} s_{N-l-1}\left(a_{1}, \ldots, \hat{a}_{i}, \ldots, a_{N}\right) \cdot A_{i}+ \\
(-1)^{N-l} s_{N-l}\left(a_{1}, \ldots, a_{N}\right) \cdot \sigma, \quad l=0, \ldots, N-1,
\end{array}
$$

where $s_{k}\left(a_{1}, \ldots, a_{N}\right)$ denotes the $k$-th elementary symmetric polynomial in the variables $a_{1}, \ldots, a_{N}$.

On the space of polynomial pencils of matrices a family of mutually compatible Poisson brackets are defined [23, 19]. They will be termed, for the sake of brevity, RSTS tensors. In a nutshell, this family can be described by saying that there is a map from degree $N$ polynomials in the variable $\lambda$ to the set of Poisson structures on the manifold of polynomial Lax matrices of the form (2.7) which sends the monomials $\lambda^{0}, \ldots, \lambda^{N}$ into $N+1$ fundamental Poisson brackets, $\Pi_{l}, l=0, \ldots, N$. In our case, the fundamental tensors $\Pi_{l}$ can be represented by matrices having the following block-diagonal structure:

$$
\Pi_{l}=\left(\begin{array}{cc}
C_{l} & 0 \\
0 & D_{l}
\end{array}\right)
$$

with:

$$
\begin{aligned}
& \left\{\begin{array}{cc}
\left(C_{l}\right)_{i j}=-\left[B_{i+j-l-1}, .\right] & i, j=1, \ldots, l \\
\left(D_{l}\right)_{i j}=\left[B_{i+j+l-1}, .\right] & i, j=1, \ldots, N-l
\end{array}\right. \\
& B_{i}=0 \text { if } i<0 \text { or } i>N, \quad \text { and } \quad B_{N}=\sigma .
\end{aligned}
$$

Lemma 1 In the "coordinates" $B_{0}, \ldots, B_{N-1}, \sigma$, the diagonal Poisson tensor $P$ (2.6) is given by the sum

$$
P=\sum_{l=0}^{N}(-1)^{N-l-1} s_{N-l}\left(a_{1}, \ldots, a_{N}\right) \Pi_{l},
$$

where the $s_{i}$ 's are the elementary symmetric polynomials in the $a_{i}$ 's, that is, it is the tensor associated with the polynomial

$$
p_{N}=\prod_{l=1}^{N}\left(\lambda-a_{i}\right)
$$


This Lemma can be proved by means of a direct computation. For the reader's convenience, we collect the main steps of it in Appendix A.

Since the Poisson tensors (2.9) form a $N+1$-parameter family of compatible Poisson tensors, we can choose as a second Poisson tensor a suitable linear combination of them to have a bihamiltonian structure on $\mathfrak{g}^{N}$. Let

$$
Q=\sum_{l=0}^{N-1}(-1)^{N-l} s_{N-l-1}\left(a_{1}, \ldots, a_{N}\right) \Pi_{l}
$$

be the tensor associated with the polynomial

$$
p_{N-1}=\left(\frac{p_{N}}{\lambda}\right)_{+}=\lambda^{N-1}-s_{1} \lambda^{N-2}+\cdots+(-1)^{N} s_{N-1} .
$$

All the integrals of motion that one can obtain from the spectral invariants of the Lax matrix (1.6) can be obtained by the GZ method applied to the pencil $Q-\lambda P$; in fact it holds (see, also, 23]):

Lemma 2 All the vector fields associated to the spectral invariants of (1.6) are bihamiltonian with respect to the pair $Q-\lambda P$.

Proof: We find convenient to work in the variables $B_{i}$. Let us define:

$$
\begin{aligned}
& K_{\alpha}^{(i)}=\operatorname{Tr}\left(\operatorname{Res}_{\lambda=0}\left(\frac{\left(\sum_{j=1}^{N} B_{j} \lambda^{j}\right)^{\alpha}}{\lambda^{i}}\right)\right) \\
& i=1, \ldots, \alpha N \quad \alpha=2, \ldots \operatorname{rk}(\mathfrak{g}) .
\end{aligned}
$$

For any fixed $\alpha$, the $\alpha N$ functions (2.13) fulfill the relations [23]:

$$
\Pi_{i} d K^{(j)}=\Pi_{i+k} d K^{(j+k)}=X^{(j-i)} .
$$

From (2.14) it follows that $X^{(i)}=0$ if $i \leq 0$ or $i>N(\alpha-1)$; in fact, if $i \leq 0$ then $K^{(i)}=0$ and $X^{(i)}=\Pi_{0} d K^{(i)}=0$, while if $i>N(\alpha-1)$, then $K^{(N+i)}=$ const and $X^{(i)}=\Pi_{N} d K^{(N+i)}=0$. Now let us set:

$$
b_{l}=(-1)^{N-l+1} s_{N-l}\left(a_{1}, \ldots, a_{N}\right),
$$

we have:

$$
\begin{aligned}
& P d K^{(j)}=\sum_{l=0}^{N} b_{l} \Pi_{l} d K^{(j)}=\sum_{l=0}^{N} b_{l} X^{(j-l)} \\
& Q d K^{(j)}=\sum_{l=1}^{N} b_{l} \Pi_{l-1} d K^{(j)}=\sum_{l=1}^{N} b_{l} X^{(j-l-1)} .
\end{aligned}
$$

Then:

$$
P d K_{\alpha}^{(j)}-Q d K_{\alpha}^{(j+1)}=b_{0} X_{\alpha}^{(j)}
$$


If one of the $a_{i}$ is equal to zero, then $b_{0}=\prod_{i=1}^{N} a_{i}=0$ and the proof is concluded. Otherwise we need to find a function $F_{\alpha}^{(j)}$ such that

$$
Q d F_{\alpha}^{(j)}=b_{0} X_{\alpha}^{(j)}
$$

We proceed by induction. If $j=1$, we have $b_{0} X_{\alpha}^{(1)}=Q \frac{b_{0}}{b_{1}} d K_{\alpha}^{(1)}$, so that $F_{\alpha}^{(1)}=$ $b_{0} / b_{1} K_{\alpha}^{(1)}$. Now let $F_{\alpha}^{(i)}$ be such that: $b_{0} X_{\alpha}^{(i)}=Q d F_{\alpha}^{(i)} \quad i=1, \ldots, j-1$. Then

$$
\begin{aligned}
& Q \frac{b_{0}}{b_{1}} d K_{\alpha}^{(j)}=b_{0} X_{\alpha}^{(j)}+\frac{b_{0} b_{2}}{b_{1}} X_{\alpha}^{(j-1)}+\cdots+\frac{b_{0} b_{N}}{b_{1}} X_{\alpha}^{(j-N+1)} \Longrightarrow \\
& \Longrightarrow b_{0} X_{\alpha}^{(j)}=Q\left(\frac{b_{0}}{b_{1}} d K_{\alpha}^{(j)}-\frac{b_{2}}{b_{1}} d F_{\alpha}^{(j-1)}-\cdots-\frac{b_{N}}{b_{1}} d F_{\alpha}^{(j-N+1)}\right) .
\end{aligned}
$$

So we have:

$$
Q d F_{\alpha}^{(j)}=b_{0} X_{\alpha}^{(j)}, \text { with } F_{\alpha}^{(j)}=\frac{b_{0}}{b_{1}} K_{\alpha}^{(j)}-\frac{1}{b_{1}} \sum_{i=1}^{N-1} b_{i+1} F_{\alpha}^{(j-i)}
$$

Some observations on the GZ sequences are in order. The starting points of the GZ sequences are given by the Casimirs of $P$. We have to distinguish two cases:

a) If $b_{0} \neq 0$, i.e. all the $a_{i}$ are different from zero, then the Casimirs of $P$ are given in terms of the spectral invariants (2.13) by the following expressions:

$$
C_{i, \alpha}=\sum_{j=1}^{\alpha N} a_{i}^{j} K_{\alpha}^{(j)} \quad i=1, \ldots, N \quad \alpha=2, \ldots \operatorname{rk}(\mathfrak{g}) .
$$

For any $\alpha$, starting the GZ sequences from suitable linear combinations of the Casimirs $C_{i, \alpha}$ we can construct $N$ GZ sequences of length $\alpha-1$ (i.e. defining $\alpha-1$ independent vector fields) each starting with a Casimir of $P$ and ending with a Casimir of $Q$.

b) If $b_{0}=0$ then only one among the $a_{i}$, say $a_{N}$, is zero. In this case equation (2.17) defines $(\operatorname{rk}(\mathfrak{g})-1)(N-1)$ independent Casimirs, instead of $(\operatorname{rk}(\mathfrak{g})-$ 1) $N$ :

$$
C_{i, \alpha}=\sum_{j=1}^{\alpha N} a_{i}^{j} K_{\alpha}^{(j)} \quad i=1, \ldots, N-1 \quad \alpha=2, \ldots \operatorname{rk}(\mathfrak{g}) .
$$

The functions (2.18) turns out to be simultaneous Casimirs for both $P$ and $Q$. The remaining $\operatorname{rk}(\mathfrak{g})-1$ Casimirs of $P$ (the rank of $P$ is obviously the same in both cases) are given by

$$
C_{N_{\alpha}}=K_{\alpha}^{(1)} \quad \alpha=2, \ldots, \operatorname{rk}(\mathfrak{g}) .
$$

To each Casimir (2.19) is associated a GZ sequence of length $(\alpha-1) N$. 


\section{The homogeneous case}

The constant term $\sigma$ in the Lax matrix (1.6) physically describes the coupling of the $i$-th spin with an "external magnetic" field $\beta_{i}=a_{i} \sigma$. The matrix $\sigma$ in the definition of the rational Lax matrix (1.6) is somewhat a free parameter in the theory. Changing $\sigma$ amounts to "changing the direction" of this magnetic field. The choice usually done in the literature is the generic one (say, $\sigma$ is a diagonal matrix with different entries); this ensures the functional independence of the coefficients of the spectral invariants of $L_{\text {rat }}$, whence the fact that they are in a sufficient number to yield complete integrability of the model.

If $\sigma$ is not generic, but the dimension of its stabilizer $\mathfrak{g}_{\sigma}:=\{\tau \in \mathfrak{g}$ s.t. $[\tau, \sigma]=$ $0\}$ is greater than the rank of $\mathfrak{g}$ the following happens. Not all the spectral invariants of the Lax matrix are functionally independent, but one can recover the "missing" integrals noticing that the functions:

$$
F_{\tau}=\operatorname{Tr}\left(\sum_{i=1}^{N} A_{i} \tau\right), \quad \tau \in \mathfrak{g}_{\sigma}
$$

commute with all the spectral invariants of $L_{\text {rat }}$.

However, something more substantial occurs for $\sigma=0$, that is, in the homogeneous case. As we have recalled in the Introduction, in such a case $H_{G}$ (1.7) defines, for $\mathfrak{g}=s l(2)$, a superintegrable Hamiltonian system and, in particular, it is possible to find another complete set of commuting first integrals which are not explicitly dependent on the parameters $a_{i}$.

From now on we will focus on this additional family of integrals, that, in the classical $N$-site $\operatorname{sl}(2)$ model can be given by the very simple formula:

$$
I_{l-1}=\sum_{j, k=1}^{l} \operatorname{Tr}\left(A_{j} \cdot A_{k}\right), l=2, \ldots, N
$$

We will introduce a further Poisson structure $R$ on the manifold $\mathfrak{g}^{N}$. As we shall show it will be possible to combine it with the diagonal Poisson structure $P$ of Eq. (2.6) to get a further Poisson pencil, not belonging to the RSTS family described in Section 2. The GZ method applied to the Poisson pencil $R-\lambda P$ will give rise to these new set of integrals. Since everything will be done in a Lie-algebraic setting, these results hold for a generic semisimple Lie algebra, and in particular, for $s l(r)$ with arbitrary $r$. 


\subsection{The additional bihamiltonian pencil}

Let us consider the bivector $R$, defined, by means of the constructions outlined in Section 2 by the following matrix:

$$
R=\left(\begin{array}{cccc}
0 & {\left[A_{1}, \cdot\right]} & \ldots & {\left[A_{1}, \cdot\right]} \\
{\left[A_{1}, \cdot\right]} & {\left[A_{2}-A_{1}, \cdot\right]} & \ldots & {\left[A_{2}, \cdot\right]} \\
\vdots & \vdots & \ddots & \vdots \\
{\left[A_{1}, \cdot\right]} & {\left[A_{2}, \cdot\right]} & \cdots & {\left[(N-1) A_{N}-\sum_{i=1}^{N-1} A_{i}, \cdot\right]}
\end{array}\right) .
$$

Proposition 3.1 The bivector $R$ defined by (3.2) is a Poisson bivector, and it is compatible with the diagonal Poisson tensor $P$.

Proof: Linearity and antisymmetry are obvious, so we must prove only the Jacobi identity. Also, we can limit ourselves to prove the assertions for the case of linear functions on $\mathfrak{g}$. If $F, G, H$ are such functions, identifying their differentials with the three $N$-tuples of matrices $\left\{\alpha_{i}\right\},\left\{\beta_{i}\right\},\left\{\gamma_{i}\right\}$, (e.g., $\frac{\partial F}{\partial A_{i}}=$ $\left.\alpha_{i}, \ldots\right)$, the Poisson bracket is defined by:

$$
\begin{gathered}
\{F, G\}_{R}=\langle d F, R d G\rangle=\sum_{i, j, k} r_{i j k} \operatorname{Tr}\left(\alpha_{i}\left[A_{k}, \beta_{j}\right]\right)=\sum_{i, j, k} r_{i j k} \operatorname{Tr}\left(A_{k}\left[\beta_{j}, \alpha_{i}\right]\right), \\
r_{i j k}=(k-1) \delta_{i j} \delta_{j k}-\theta_{(i-k)} \delta_{i j}+\theta_{(j-i)} \delta_{i k}+\theta_{(i-j)} \delta_{j k}
\end{gathered}
$$

where $\delta$ is the usual Kronecker symbol and $\theta$ is the discrete Heaviside function, normalized as

$$
\theta_{(i)}= \begin{cases}1 & \text { if } i>0 \\ 0 & \text { if } i \leq 0\end{cases}
$$

The Jacobi identity reads

$$
\begin{gathered}
\left\{H,\{F, G\}_{R}\right\}_{R}+\left\{F,\{G, H\}_{R}\right\}_{R}+\left\{G,\{H, F\}_{R}\right\}_{R}= \\
\sum_{i, j, k, l, m} r_{i j k} r_{l m j}\left(\operatorname{Tr}\left(A_{k}\left[\left[\beta_{m}, \alpha_{l}\right], \gamma_{i}\right]\right)+\operatorname{Tr}\left(A_{k}\left[\left[\alpha_{m}, \gamma_{l}\right], \beta_{i}\right]\right)+\operatorname{Tr}\left(A_{k}\left[\left[\gamma_{m}, \beta_{l}\right], \alpha_{i}\right]\right)\right),
\end{gathered}
$$

which, renaming the indices, becomes

$$
\begin{aligned}
& \sum_{i, j, k, l, m} r_{i j k} r_{l m j} \operatorname{Tr}\left(A_{k}\left[\left[\beta_{m}, \alpha_{l}\right], \gamma_{i}\right]\right)+r_{m j k} r_{i l j} \operatorname{Tr}\left(A_{k}\left[\left[\alpha_{l}, \gamma_{i}\right], \beta_{m}\right]\right)+ \\
& \quad+r_{l j k} r_{m i j} \operatorname{Tr}\left(A_{k}\left[\left[\gamma_{i}, \beta_{m}\right], \alpha_{l}\right]\right) .
\end{aligned}
$$

A sufficient condition for the last expression to be zero is that for any $k$ it holds:

$$
\sum_{j} r_{i j k} r_{l m j}=\sum_{j} r_{m j k} r_{i l j}
$$


In fact, a consequence of (3.3) to hold is that implies that $t_{i k l m}=\sum_{j} r_{i j k} r_{l m j}$ is invariant for cyclic permutations of the indices $i, l, m$. So, if (3.3) holds we can write:

$$
\begin{aligned}
& \left\{H,\{F, G\}_{R}\right\}_{R}+\left\{F,\{G, H\}_{R}\right\}_{R}+\left\{G,\{H, F\}_{R}\right\}_{R}= \\
& =\sum_{i, k, l, m} t_{i k l m} \operatorname{Tr}\left(A_{k}\left(\left[\left[\beta_{m}, \alpha_{l}\right], \gamma_{i}\right]+\left[\left[\alpha_{l}, \gamma_{i}\right], \beta_{m}\right]+\left[\left[\gamma_{i}, \beta_{m}\right], \alpha_{l}\right]\right)\right),
\end{aligned}
$$

which vanishes thanks to the Jacobi identity in $\mathfrak{g}$.

Let us show that (3.3) holds in our case. By means of algebraic manipulations, namely cycling through $i, l, m$ and renaming the indices using the Kronecker's $\delta$, we obtain:

$$
\begin{aligned}
& \sum_{j} r_{i j k} r_{l m j}-\sum_{j} r_{m j k} r_{i l j}= \\
& =\delta_{i k} \delta_{l m}\left[(l-i) \theta_{(l-i)} \sum_{j} \theta_{(j-i)} \theta_{(l-j)}\right]+\delta_{i l} \delta_{m k}\left[(i-m) \theta_{(i-m)}+\sum_{j} \theta_{(j-m)} \theta_{(i-j)}\right]+ \\
& +\delta_{l m}\left[\theta_{(i-k)}\left(\theta_{(l-i)}-\theta_{(l-k)}\right)+\theta_{(l-k)} \theta_{(i-l)}\right]+\delta_{i l}\left[\theta_{(m-k)}\left(\theta_{(i-k)}-\theta_{(i-m)}\right)-\theta_{(i-k)} \theta_{(m-i)}\right]+ \\
& +\delta_{i k}\left[\theta_{(l-i)}\left(\theta_{(m-l)}-\theta_{(m-i)}\right)+\theta_{(m-i)} \theta_{(l-m)}\right]+\delta_{m k}\left[\theta_{(l-m)}\left(\theta_{(i-m)}-\theta_{(i-l)}\right)-\theta_{(i-m)} \theta_{(l-i)}\right]
\end{aligned}
$$

Using the identities:

$$
\begin{aligned}
& \sum_{j} \theta_{(j-i)} \theta_{(l-j)}=(l-i-1) \theta_{(l-i)} \\
& \theta_{(i-k)}\left(\theta_{(l-i)}-\theta_{(l-k)}\right)+\theta_{(l-k)} \theta_{(i-l)}=-\theta_{(l-k)} \delta_{i l}
\end{aligned}
$$

we see that every term cancels out.

We now prove that $R$ is compatible with the diagonal tensor $P$. The characteristic condition for the compatibility of two Poisson tensors is

$$
\left\{H,\{F, G\}_{P}\right\}_{R}+\left\{H,\{F, G\}_{R}\right\}_{P}+\text { cyclic permutations of } F, G, H=0 \text {. }
$$

Recalling that $\{F, G\}_{P}=\sum_{i, j, k} \delta_{i j} \delta_{j k} \operatorname{Tr}\left(A_{k}\left[\frac{\partial G}{\partial A_{j}}, \frac{\partial F}{\partial A_{i}}\right]\right)$, one shows that a sufficient condition for the compatibility of $R$ and $P$ is that the quantity

$$
s_{i k l m}=\sum_{j}\left(r_{i j k} \delta_{l m} \delta_{m j}+\delta_{i j} \delta_{j k} r_{l m j}\right)
$$

be invariant under cyclic permutations of the indices $i, l, m$ for all $k$ 's. Actually,

$$
s_{i k l m}=(k+i) \delta_{i k} \delta_{l m} \delta_{i l}-\theta_{(i-k)} \delta_{l m} \delta_{i l}+\theta_{(i-m)} \delta_{l m} \delta_{k l}+\theta_{(m-l)} \delta_{i k} \delta_{i l}+\theta_{(l-m)} \delta_{i k} \delta_{i m}
$$

that manifestly satisfies this property. 
Remark. By the previous proposition, we can endow, for every $N$, the space $\left(\mathfrak{g}^{*}\right)^{N}$ with a bihamiltonian structure $P_{\lambda}=R-\lambda P$. A natural question arises, that is what is the connection with the RSTS family of Poisson structures discussed in Section 2, We do not have yet a complete answer to this point; however, as we will show in Appendix $\mathrm{B}$, the new tensor $R$ is not compatible with the generic element of the RSTS family (2.9).

\subsection{The Lenard Chains}

We construct the Lenard chains for the Poisson pencil $R-\lambda P$, using the GZ recipe discussed in Section 2, To shorten notations we define:

$$
\begin{aligned}
& B_{l}=\sum_{i=1}^{l-1} A_{i}, \quad F_{\beta, l}^{(\alpha)}=\operatorname{res}_{\lambda=0} \frac{1}{\lambda^{\alpha+1}}\left(\lambda A_{l}+B_{l}\right)^{\beta} \\
& H_{\beta, l}^{(\alpha)}=\operatorname{Tr}\left(F_{\beta, l}^{(\alpha)}\right) .
\end{aligned}
$$

We will first find a kind of "modified" recursion relation.

Lemma 3 It holds:

$$
P d H_{\beta, l}^{(\alpha-1)}=(R-(l-2) P) d H_{\beta, l}^{(\alpha)}
$$

for $\alpha=1, \ldots, \beta$

Proof: We proceed by induction on $\beta$. If $\beta=2$ we have:

$$
\begin{aligned}
& H_{2, l}^{(0)}=\operatorname{tr}\left(B_{l}^{2}\right) \quad \Rightarrow \quad \frac{\partial H_{2, l}^{(0)}}{\partial A_{j}}=2 \theta_{(l-j)} B_{l} \\
& H_{2, l}^{(1)}=2 \operatorname{tr}\left(A_{l} B_{l}\right) \quad \Rightarrow \quad \frac{\partial H_{2, l}^{(1)}}{\partial A_{j}}=2 \theta_{(l-j)} A_{l}+B_{l} \delta_{j l} \\
& H_{2, l}^{(2)}=\operatorname{tr}\left(A_{l}^{2}\right) \quad \Rightarrow \quad \frac{\partial H_{2, l}^{(2)}}{\partial A_{j}}=2 A_{l} \delta_{j l} .
\end{aligned}
$$

By direct computation we obtain:

$$
\begin{aligned}
& \left((R-(l-2) P) d H_{2, l}^{(2)}\right)_{i}=2\left(\theta_{(l-i)}\left[A_{i}, A_{l}\right]+\delta_{i l}\left[A_{i}, B_{i}\right]\right)=\left(P d H_{2, l}^{(1)}\right)_{i} \\
& \left((R-(l-2) P) d H_{2, l}^{(1)}\right)_{i}=2 \theta_{(l-i)}\left[A_{i}, B_{l}\right]=\left(P d H_{2, l}^{(0)}\right)_{i}
\end{aligned}
$$


We use the inductive hypothesis in the case $\alpha \leq \beta-1$. Plugging in the following identities:

$$
\begin{aligned}
& {\left[B_{l}, F_{\beta-1, l}^{(\alpha)}\right]+\left[A_{l}, F_{\beta-1, l}^{(\alpha-1)}\right]=0} \\
& \frac{\partial H_{\beta, l}^{(\alpha)}}{\partial A_{j}}=\theta_{(l-j)} \frac{\partial H_{\beta, l}^{(\alpha)}}{\partial B_{l}}+\delta_{j l} \frac{\partial H_{\beta, l}^{(\alpha)}}{\partial A_{l}} \\
& \frac{\partial H_{\beta, l}^{(\alpha)}}{\partial A_{j}}=\theta_{(l-j)} F_{\beta-1, l}^{(\alpha)}+B_{l} \frac{\partial H_{\beta-1, l}^{(\alpha)}}{\partial A_{j}}+\delta_{j l} F_{\beta-1, l}^{(\alpha-1)}+A_{l} \frac{\partial H_{\beta-1, l}^{(\alpha-1)}}{\partial A_{j}} \\
& \frac{\partial H_{\beta, l}^{(\alpha)}}{\partial A_{l}}=\frac{\partial H_{\beta, l}^{(\alpha-1)}}{\partial B_{l}}
\end{aligned}
$$

and using the inductive hypothesis, one obtains by straightforward computation

$$
P d H_{\beta, l}^{(\alpha-1)}-(R-(l-2) P) d H_{\beta, l}^{(\alpha)}=0 .
$$

The case $\alpha=\beta$ can be easily verified by direct computation.

Notice that identities (3.7) and (3.8) follow from (3.4) and (3.5). The identity (3.9) follows from the recursive formula for $F_{\beta, l}^{(\alpha)}$ :

$$
\begin{aligned}
& F_{\beta, l}^{(\alpha)}=B_{l} F_{\beta-1, l}^{(\alpha)}+A_{l} F_{\beta-1, l}^{(\alpha-1)} \\
& F_{0, l}^{(0)}=1 \\
& F_{\beta, l}^{(\alpha)}=0 \quad \text { if } \alpha>\beta \text { or } \alpha<0,
\end{aligned}
$$

while (3.10) can be proven again by induction. For $\alpha=2$ it holds:

$$
\frac{\partial H_{2, l}^{(2)}}{\partial A_{l}}=2 A_{l}=\frac{\partial H_{2, l}^{(1)}}{\partial B_{l}} \quad \frac{\partial H_{2, l}^{(1)}}{\partial A_{l}}=2 B_{l}=\frac{\partial H_{2, l}^{(0)}}{\partial B_{l}}
$$

Then, if $\alpha \leq \beta-1$ we can use the inductive hypothesis and get:

$$
\frac{\partial H_{\beta, l}^{(\alpha)}}{\partial A_{l}}-\frac{\partial H_{\beta, l}^{(\alpha-1)}}{\partial B_{l}}=B_{l}\left(\frac{\partial H_{\beta-1, l}^{(\alpha)}}{\partial A_{l}}-\frac{\partial H_{\beta-1, l}^{(\alpha-1)}}{\partial B_{l}}\right)+A_{l}\left(\frac{\partial H_{\beta-1, l}^{(\alpha-1)}}{\partial A_{l}}-\frac{\partial H_{\beta-1, l}^{(\alpha-2)}}{\partial B_{l}}\right)=0 .
$$

The case $\alpha=\beta$ is again a matter of simple computation.

\section{Proposition 3.2 The Hamiltonians}

$$
\begin{aligned}
& K_{\beta, l}^{(\beta-k)}=\sum_{j=0}^{k-1}\left(\begin{array}{c}
k-1 \\
j
\end{array}\right)(l-2)^{k-j-1} H_{\beta, l}^{(\beta-j-1)} \\
& K_{\beta, l}^{(\beta)}=H_{\beta, l}^{(\beta)}, \quad l=2, \ldots, N
\end{aligned}
$$

satisfy the standard Lenard-Magri relations $P d K_{\beta, l}^{(\alpha-1)}=R d K_{\beta, l}^{(\alpha)}, \quad \alpha=1, \ldots \beta, \forall \beta$. 
Proof: Using Lemma 3, we have:

$$
\begin{aligned}
& \operatorname{Rd} K_{\beta, l}^{(\beta-k)}= \\
= & \operatorname{Pd}\left(\sum_{j=0}^{k-1}\left(\begin{array}{c}
k-1 \\
j
\end{array}\right)\left((l-2)^{k-j-1} H_{\beta, l}^{(\beta-j-2)}+(l-2)^{k-j} H_{\beta, l}^{(\beta-j-1)}\right)\right)= \\
= & \operatorname{Pd}\left(K_{\beta, l}^{(\beta-k-1)}+\sum_{j=1}^{k-1}\left[\left(\begin{array}{c}
k-1 \\
j-1
\end{array}\right)+\left(\begin{array}{c}
k-1 \\
j
\end{array}\right)\right](l-2)^{k-j} H_{\beta, l}^{(\beta-j-1)}\right)= \\
= & \operatorname{Pd}\left(\sum_{j=0}^{k}\left(\begin{array}{c}
k \\
j
\end{array}\right)(l-2)^{k-j} H_{\beta, l}^{(\beta-j-1)}\right)=P d K_{\beta, l}^{(\beta-k-1)}
\end{aligned}
$$

\subsection{Complete integrability for $\mathfrak{g}=\mathrm{sl}(\mathbf{r})$}

We now prove that the Hamiltonians (3.5) together with additional integrals one can recover from the global $S L(r)$ invariance of the model provide complete integrability in the case $\mathfrak{g}=s l(r)$. We start by remarking that the content of Lemma 3 and Proposition 3.2 can be rephrased as follow: If we introduce the $N$ matrices:

$$
L_{1}=A_{1}, \quad L_{a}=\lambda A_{a}+B_{a}, \quad a=2, \ldots, N
$$

then they evolve isospectrally along any of the vector field of the hierarchy, that is, (since the matrices $A_{i}$ are generically simple) along Lax type equations.

The dimension of the manifold $M=\operatorname{sl}(r)^{N}$ is $d_{M}=\left(r^{2}-1\right) N$, and the dimension of the generic symplectic leaf of $P$ is $d_{S}=d_{M}-N(r-1)=r(r-1) N$. We notice that the we recover (as expected) all the Casimirs of $P$ considering: a) the spectral invariants of $L_{1}=A_{1}$ (this gives $N-1$ common Casimirs), and b) the higher order terms in expansions of $L_{l}: H_{\alpha, l}^{(\alpha)}, \alpha=2, \ldots, r, l=2, \ldots, N$. Since it holds $H_{\beta, l}^{(0)}=\sum_{k=0}^{\beta} H_{\beta, l-1}^{(k)}$, we consider the set

$$
H_{\beta, l}^{(\alpha)} \quad \beta=2, \ldots, r \quad \alpha=1, \ldots, \beta-1
$$

This provides us with a distinguished sequence of $\frac{r(r-1)}{2}$ mutually commuting Hamiltonians. Clearly, if $l \neq l^{\prime}$, the sets $\left\{H_{\beta, l}^{(\alpha)}\right\}$ and $\left\{H_{\beta, l^{\prime}}^{(\alpha)}\right\}$ are functionally independent, since they depend on a different set of variables. So, the counting of the number of independent Hamiltonians boils down to compute the counting of independent coefficients in the determinant

$$
\operatorname{det}(\mu-\lambda A+B), A, B \in \operatorname{sl}(r) .
$$


This problem was solved in [6], (see, also, [1]) and, actually, the number is exactly $\frac{r(r-1)}{2}$. Hence, the Lenard sequences associated with $R-\lambda P$ provide us with a total number of $(N-1) \frac{r(r-1)}{2}$ commuting Hamiltonian, plus the $N(r-1)$ Casimirs. For complete integrability we are missing $r(r-1) / 2$ more commuting integrals.

They are associated with the global $S L(r)$ invariance of the problem, and, in the bihamiltonian picture, can be described as follows. For every $\tau \in \operatorname{sl}(r)$ we can consider the linear function

$$
H_{\tau}=\operatorname{tr}\left(\sum_{i=1}^{N} A_{i} \tau\right) .
$$

The Lenard "sequence" associated with such functions is somewhat peculiar; indeed, since $R d H_{\tau}=(N-1) P d H_{\tau}$, we can associate to each $H_{\tau}$ a Lenard diagram which is (up to a constant) a closed loop, to be compared with the usual ladder typical of iterable Hamiltonians. However, the usual argument of bihamiltonian recurrence, shows that, for any $\tau$,

$$
\left\{K_{\beta, l}^{(\alpha)}, H_{\tau}\right\}_{P}=\left\{K_{\beta, l}^{(\alpha)}, H_{\tau}\right\}_{R}=0, \forall \alpha, \beta, l .
$$

Indeed, one has, e.g., the equality:

$$
\left\{K_{\beta, l}^{(\alpha)}, H_{\tau}\right\}_{P}=\left\{K_{\beta, l}^{(\alpha+1)}, H_{\tau}\right\}_{R}=(N-1) \cdot\left\{K_{\beta, l}^{(\alpha+1)}, H_{\tau}\right\}_{P} .
$$

This argument shows how to recover, in the bihamiltonian formalism, the integrals associated with the global $S L(r)$ invariance of the model. Clearly, this family of $r^{2}-1$ integrals is not a commutative one.

To recover the maximal Abelian subalgebra inside the Poisson algebra generated by the functions $H_{\tau}$, one can consider (see, e.g., [5]):

a) The $r-1$ independent elements $H_{h_{1}}, \ldots, H_{h_{r-1}}$ associated with, say, the standard Cartan subalgebra of $\operatorname{sl}(r)$;

b) The Gel'fand-Cetlin invariants, that is, the Casimirs of the nested subalgebras

$$
\operatorname{sl}(2) \subset \operatorname{sl}(3) \subset \cdots \subset \operatorname{sl}(r)
$$

under the map $\operatorname{sl}(r)^{N} \rightarrow \operatorname{sl}(r)$ sending the $N$-tuple $\left\{A_{1}, \ldots, A_{N}\right\}$ into the total sum, $A_{\text {tot }}=\sum_{i=1}^{N} A_{i}$.

Noticing that the Gel'fand-Cetlin functions corresponding to the last element of the chain (3.12) are given by $\sum_{k=0}^{\beta} H_{\beta, N}^{(k)}$, we obtain $r-1+\sum_{i=2}^{r-1}(i-1)=\frac{r(r-1)}{2}$ additional commuting integrals, which is exactly the number of commuting integrals we were looking for to insure complete integrability of the model.

We end this Section with a comment concerning super-integrability of the model. To this end we remark that we have at our disposal two Poisson pencils 
to construct families of commuting integrals for the Gaudin (homogeneous) Hamiltonian $H_{G}$ : the pencil $R-\lambda P$ and the pencil $Q-\lambda P$, described in Section 21. On the $d_{N, r}=N(r(r-1))$-dimensional generic symplectic leaves of $P$ they give rise to two distinct $\left.d_{N, r} / 2\right)$ families of integrals of the motion $K_{m}^{l j}$ and $\widetilde{K}_{m}^{l j}$. Direct computations (which we performed for $r=3,4$ and $N \leq 6$ ) suggest that the number of functionally independent elements in the union of the two families be $d_{N, r}-(r-1)$. In other words, also taking into account the integrals coming from the global $S L(r)$ invariance of the model, we have super-integrability for the $\operatorname{sl}(r)$ Gaudin model, that, however, is maximal only for the $\operatorname{sl}(2)$ case.

\section{Separation of Variables for the $\mathrm{sl}(2)$ case}

We consider now the $N$-particle $s l(2)$ case. The aim is to show that the Hamilton-Jacobi equations associated with the Hamiltonians

$$
H_{a}=\operatorname{Tr}\left(A_{i} \cdot \sum_{j=1}^{a-1} A_{j}\right), \quad a=2, \ldots, N
$$

and, in particular, the $\mathrm{H}-\mathrm{J}$ equations associated with the physical Hamiltonian $H_{G}=\frac{1}{2} \sum_{i=1}^{N} H_{i}$ are separable in a very "simple" set of coordinates. Our analysis is based on the so-called bihamiltonian scheme for SoV, recently introduced in the literature (see, e.g., [21, 2, 1]). In particular, we will use the results for systems with an arbitrary number of (anchored) Lenard chain exposed in 9].

We consider the manifold $M=\operatorname{sl}(2)^{N}$, endowed with the Poisson pencil $R-\lambda P$, explicitly parametrized with the $N$ matrices

$$
A_{i}=\left[\begin{array}{cc}
h_{i} & f_{i} \\
e_{i} & -h_{i}
\end{array}\right] .
$$

The generic symplectic leaf $S$ of $P$ is a $2 N$ dimensional symplectic manifold, defined by the equations

$$
C_{i}=\frac{1}{2} \operatorname{Tr} A_{i}^{2}=h_{i}^{2}+e_{i} f_{i}, \quad i=1, \ldots, N,
$$

and can be (generically) endowed with the $2 N$ coordinates $\left\{h_{i}, f_{i}\right\}_{i=1, \ldots, N}$.

According to the bihamiltonian scheme [8], one modifies the tensor $R$ in order to obtain a second compatible structure on $S$. Let us define the $N$ vector fields

$$
Z_{i}=\frac{1}{f_{i}} \frac{\partial}{\partial e_{i}}
$$

\footnotetext{
${ }^{1}$ With the proviso in mind that one has to set $\sigma=0$ in those formulas
} 
and, for any pair of functions $F, G$ on $M$, the following brackets:

$$
\{F, G\}_{R^{\prime}}=\{F, G\}_{R}-\sum_{a=2}^{N}\left(\left\{F, H_{a}\right\}_{P} Z_{a}(G)-\left\{G, H_{a}\right\}_{P} Z_{a}(F)\right),
$$

where $X(F)$ denotes the Lie derivative of $F$ with respect to the vector field $X$.

Thanks to the specific form of the vector fields $Z_{a}$, these new brackets restrict to $S$ and give rise to Poisson brackets on $S$, which are compatible with the ones naturally induced by $P$. So, $S$ is a symplectic manifold with respect to the restriction of the brackets associated with $P$, and is endowed with a $(1,1)$ tensor $\mathcal{N}$, with vanishing Nijenhuis torsion defined by

$$
\mathcal{N}=R^{\prime} \circ P^{-1}
$$

In such a geometrical setting, the bihamiltonian scheme for SoV considers sets of coordinates $\left\{u_{i}, v_{i}\right\}$ (called Nijenhuis coordinates) associated with the eigenvalues $\lambda_{i}$ of $\mathcal{N}$, characterized by the equations:

$$
\mathcal{N}^{*} d u_{i}=\lambda_{i} d u_{i}, \quad \mathcal{N}^{*} d v_{i}=\lambda_{i} d v_{i}
$$

whose Poisson brackets attain the remarkable form [20, 14]:

$$
\begin{aligned}
& \left\{u_{i}, u_{j}\right\}_{P}=\left\{u_{i}, u_{j}\right\}_{R^{\prime}}=\left\{v_{i}, v_{j}\right\}_{P}=\left\{v_{i}, v_{j}\right\}_{R^{\prime}}=0 \\
& \left\{u_{i}, v_{j}\right\}_{P}=\delta_{i j} \vartheta_{i}\left(u_{i}, v_{i}\right), \quad\left\{u_{i}, v_{j}\right\}_{R^{\prime}}=\lambda_{i}\left\{u_{i}, v_{j}\right\}_{P}
\end{aligned}
$$

for some functions $\vartheta_{i}\left(u_{i}, v_{i}\right)$.

We shall prove the above statements directly displaying a set of Nijenhuis coordinates on the symplectic leaves of $P$.

Proposition 4.1 The $2 N$ functions

$$
\begin{array}{ll}
\lambda_{1}=\sum_{i=1}^{N} f_{i}, \quad \lambda_{a}=-\frac{\sum_{k=1}^{a-1} f_{k}}{f_{a}}+(a-2), \quad a=2, \ldots, N \\
\mu_{1}=\sum_{i=1}^{N} h_{i}, \quad \mu_{a}=\left(\lambda_{a}-(a-2)\right) h_{a}+\sum_{k=1}^{a-1} h_{k}, \quad a=2, \ldots, N
\end{array}
$$

provide a set of Nijenhuis coordinates on $S$. In particular the $\lambda_{a}, a=2, \ldots, N$ are the non-vanishing eigenvalues of $\mathcal{N}^{*}$, while $\lambda_{1}$ and $\mu_{1}$ span its (2-dimensional) kernel.

Proof. According to Equation (4.4) and the definition of the Nijenhuis tensor $\mathcal{N}$, noticing that both $P$ and $R^{\prime}$ restrict to $S$ and that $Z_{a}\left(\lambda_{i}\right)=Z_{a}\left(\mu_{i}\right)=0$ for 
$a=2, \ldots, N, i=1, \ldots, N$, we have to show that, for any coordinate $x_{i}$, that is, $\left.x_{i}=e_{i}, h_{i}, f_{i}, i=1, \ldots, N\right)$ it holds

$$
\begin{aligned}
& \left\{x_{i}, \lambda_{1}\right\}_{R}-\sum_{a=2}^{N}\left\{H_{a}, \lambda_{1}\right\}_{P} Z_{a}\left(x_{i}\right)=0 \\
& \left\{x_{i}, \lambda_{b}\right\}_{R}-\sum_{a=2}^{N}\left\{H_{a}, \lambda_{b}\right\}_{P} Z_{a}\left(x_{i}\right)-\lambda_{b}\left\{x_{i}, \lambda_{b}\right\}_{P}, b=2, \ldots, N
\end{aligned}
$$

as well as

$$
\begin{aligned}
& \left\{x_{i}, \mu_{1}\right\}_{R}-\sum_{a=2}^{N}\left\{H_{a}, \mu_{1}\right\}_{P} Z_{a}\left(x_{i}\right)=0 \\
& \left\{x_{i}, \mu_{b}\right\}_{R}-\sum_{a=2}^{N}\left\{H_{a}, \mu_{b}\right\}_{P} Z_{a}\left(x_{i}\right)=\lambda_{b}\left\{x_{i}, \mu_{b}\right\}_{P}, b=2, \ldots, N .
\end{aligned}
$$

The proof that these equations holds true is a matter of direct computations. One simply has to plug the explicit expressions of the Poisson brackets

$$
\begin{aligned}
& \left\{h_{i}, e_{j}\right\}_{P}=\delta_{i j} e_{j}, \quad\left\{h_{i}, f_{j}\right\}_{P}=-\delta_{i j} f_{j}, \quad\left\{e_{i}, f_{j}\right\}_{P}=2 \delta_{i j} h_{j} \\
& \left\{h_{i}, e_{j}\right\}_{R}=\delta_{i j}\left[(i-1) e_{i}-\sum_{k=1}^{i-1} e_{k}\right]+\theta_{(i-j)} e_{j}+\theta_{(j-i)} e_{i} \\
& \left\{h_{i}, e_{j}\right\}_{R}=-\delta_{i j}\left[(i-1) f_{i}-\sum_{k=1}^{i-1} f_{k}\right]-\theta_{(i-j)} f_{j}-\theta_{(j-i)} f_{i} \\
& \left\{e_{i}, f_{j}\right\}_{R}=2\left\{\delta_{i j}\left[(i-1) h_{i}-\sum_{k=1}^{i-1} h_{k}\right]+\theta_{(i-j)} h_{j}+\theta_{(j-i)} h_{i}\right\} .
\end{aligned}
$$

into equations (4.8) and (4.9), and use the identities

$$
\begin{aligned}
& \sum_{j=1}^{n-1} \delta_{i j}=\theta_{(n-i)}, \quad \sum_{j=1}^{n-1} \theta_{(j-i)} F_{j}=\theta_{(n-i)} \sum_{j=i+1}^{n-1} F_{j} \\
& \sum_{j=1}^{n-1} \theta_{(i-j)} F_{j}=\left(\theta_{(i-n)}+\delta_{i n}\right) \sum_{j=1}^{n-1} F_{j}+\theta_{(n-i)} \sum_{j=1}^{i-1} F_{j} .
\end{aligned}
$$

For example, let us consider $x_{i} \equiv h_{i}$. Since $Z_{a}\left(h_{i}\right)=0$, we have (for $\left.n \geq 2\right)$ :

$$
\left\{h_{i}, \lambda_{n}\right\}_{P}=\left(\lambda_{n}-n+2\right) \delta_{i n}+\theta_{(n-i)} \frac{f_{i}}{f_{n}},
$$


and

$$
\begin{aligned}
& \left\{h_{i}, \lambda_{n}\right\}_{R}=\frac{1}{f_{n}} \sum_{j=1}^{n-1}\left\{\delta_{i j}\left[(i-1) f_{i}-\sum_{k=1}^{i-1} f_{k}\right]+\theta_{(i-j)} f_{j}+\theta_{(j-i)} f_{i}\right\}+ \\
& +\frac{\lambda_{n}-n+2}{f_{n}}\left\{\delta_{i n}\left[(i-1) f_{i}-\sum_{k=1}^{i-1} f_{k}\right]+\theta_{(i-n)} f_{n}+\theta_{(n-i)} f_{i}\right\}= \\
& =\lambda_{n}\left(\delta_{i n}\left(\lambda_{n}-n+2\right)+\theta_{(n-i)} \frac{f_{i}}{f_{n}}\right)
\end{aligned}
$$

thanks to identities (4.10), (4.11).

The other cases of equations (4.84.9) are proved with similar computations.

To construct a set of canonical Nijenhuis coordinates (usually considered in the bihamiltonian scheme for SoV and quite naturally termed Darboux-Nijenhuis coordinates) $\left\{\lambda_{i}, \phi_{i}\right\}_{i=1, \ldots, N}$ from the Nijenhuis coordinates $\left\{\lambda_{i}, \mu_{i}\right\}_{i=1, \ldots, N}$ one notices that an explicit computation gives:

$$
\left\{\lambda_{1}, \mu_{1}\right\}=-\lambda_{1}, \quad\left\{\lambda_{a}, \mu_{a}\right\}=\left(\lambda_{a}-(a-2)\right)\left(\lambda_{a}-(a-1)\right), a=2, \ldots, N .
$$

Indeed, the first equation is trivially verified; for the remaining set of $N-1$ relations one has

$$
\begin{aligned}
& \left\{\mu_{n}, \lambda_{n}\right\}=-\frac{1}{f_{n}}\left\{\sum_{i=1}^{n-1} h_{i}, \sum_{i=1}^{n-1} f_{i}\right\}-\left(\lambda_{n}-(n-2)\right)\left\{h_{n}, 1 / f_{n}\right\} \sum_{i=1}^{n-1} f_{i}= \\
& =\frac{\sum_{i=1}^{n-1} f_{i}}{f_{n}}-\left(\lambda_{n}-(n-2)\right) \frac{\sum_{i=1}^{n-1} f_{i}}{f_{n}}=\left(\lambda_{n}-(n-2)\right)\left(\lambda_{n}-(n-1)\right) .
\end{aligned}
$$

Hence, one can choose

$$
\phi_{1}=-\frac{\sum_{i=1}^{N} h_{i}}{\sum_{i=1}^{N} f_{i}}, \quad \phi_{a}=\frac{\mu_{a}}{\left(\lambda_{a}-(a-2)\right)\left(\lambda_{a}-(a-1)\right)} \quad a=2, \ldots, N
$$

to have, together with the $\lambda_{i}$ a set of Darboux-Nijenhuis coordinates.

To find the separation relations, we make contact with the so-called Sklyanin magic recipe [27]. To this end, we modify the Lax matrices (3.11), by a suitable shift in the spectral parameter $\lambda$. Namely we define

$$
\widetilde{L}_{1}=L_{1} ; \quad \widetilde{L}_{a}=(\lambda-(a-2)) A_{a}+\sum_{b=1}^{a-1} A_{b}, \quad a=2, \ldots, N .
$$

We notice that the spectral invariants of $\widetilde{L_{a}}$ are combinations of the Hamiltonians $H_{\beta, l}^{(\alpha)}$ and of the common Casimirs we considered in Section 3.3, and 
provide an equivalent set of involutive constants of the motion (together with $\left.H_{1}=\sum h_{i}\right)$.

As one can easily notice, the Nijenhuis coordinates $\lambda_{a}, a=2, \ldots, N$ are nothing but the zeroes of the $(1,2)$ entry of the Lax matrix $\widetilde{L}_{a}$ of eq. (4.14), while the Nijenhuis coordinates $\mu_{a}, a=2, \ldots, N$ are the values for $\lambda=\lambda_{a}$ of the $(1,1)$ entry of the same matrix. Taking into account that $\mu_{1}$ is the first Hamiltonian $H_{1}$, we see that the Nijenhuis coordinates, the Hamiltonians and the Casimirs $C_{i}=\operatorname{Tr}\left(A_{i}\right)^{2}$ are related by the separated equations:

$$
\mu_{1}-H_{1}=0 ; \quad \operatorname{Det}\left(\mu_{a}-\left(\left(\lambda_{a}-(a-2)\right) A_{a}+\sum_{i=1}^{a-1} A_{i}\right)\right)=0
$$

whence one can directly find, using the relations (4.13), the "canonical" separation relations for the Hamilton-Jacobi equation associated with the $\operatorname{sl}(2)$ Gaudin Hamiltonians. We notice that these are quadratic equations in the separated coordinates, and hence explicitly solvable by elementary functions, for every number of sites $N$.

\section{Summary of the Results}

In this paper we have used tools from bihamiltonian geometry to study the integrability of (rational XXX) Gaudin models, associated with the Lie algebra $s l(r)$. We first framed the general (that is, inhomogeneous) model within the Gel'fand-Zakharevich scheme by selecting a suitable pencil of Poisson brackets induced by a natural family of Poisson brackets on the space of matrix polynomials.

Then we extensively studied the homogeneous case. We considered an alternative complete set of mutually commuting constants of the motion $I_{k}$ (independent of the parameters usually entering the formulation of Gaudin models). These integrals (actually, a subfamily thereof), in the $s u(2)$ case, coincide with the Hamiltonians of the bending flows of Kapovich and Millson on the moduli space of polygons in the Euclidean space.

We introduced an "additional" Poisson tensor which forms, together with the standard Lie-Poisson tensor, a bihamiltonian pencil. The GZ analysis of such a bihamiltonian structure provides exactly the alternative set of constants of the motion $I_{k}$ for the $s l(2)$ case. By using such a bihamiltonian scheme, we extended this analysis to the $s l(r)$ case; in particular, we show that the higher rank counterparts of the additional set of integrals guarantee complete integrability of the $s l(r)$-Gaudin magnet. Since, we still have at our disposal the Jurčo-Sklyanin integrals, we conclude that the Gaudin magnet is superintegrable (although we could not establish maximal super-integrability) also in the $\operatorname{sl}(r)$ case. 
We furthermore have explicitly shown in the $\operatorname{sl}(2)$ case that the HamiltonJacobi equations associated with the set of additional integrals can be solved by separation of variables, using the bihamiltonian scheme for SoV which has recently been considered in the literature. Actually, what we found is a set of separation coordinates alternative to the "standard" one found by Sklyanin and the "Montreal group", based on the standard Lax representation for the (homogeneous) Gaudin model. This should not be regarded as a surprise, since it is well known that superintegrability is related with the existence of different sets of separation coordinates. In this set of coordinates, the H-J equations can be explicitly solved by elementary functions, or, otherwise stated, the separation coordinates (for the $s l(2)$-magnet) live on genus 0 spectral curves for any number of particles, while, in the "standard picture", the genus of the spectral curve grows linearly with $N$.

\section{Acknowledgments}

We wish to thank J. Harnad for many useful discussions, and, especially, for drawing our attention to the papers [17, 11] concerning the bending flows. Also, we thank B. Dubrovin, F. Magri, M. Pedroni, and O. Ragnisco for their interest in this work.

\section{Appendix A}

In this appendix we sketch the proof of Lemma 1. Actually we will prove the converse statement, i.e., that under the map (2.8) the Poisson tensor (2.11) is sent exactly in the diagonal Poisson tensor (2.6).

We denote with $J$ the Jacobian of the transformation:

$$
J_{i j}=\frac{\partial B_{i-1}}{\partial A_{j}}=(-1)^{N-i} s_{N-i}\left(a_{1}, \ldots, \hat{a_{j}}, \ldots, a_{N}\right) .
$$

Using the identity:

$$
\sum_{j=1}^{N} x^{j-1}(-1)^{N-j} s_{N-j}\left(a_{1}, \ldots, \hat{a_{k}}, \ldots, a_{N}\right)=\prod_{l \neq k}\left(x-a_{k}\right)
$$

the inverse matrix of A.1 is easily obtained:

$$
\left(J^{-1}\right)_{i j}=\frac{a_{i}^{j-1}}{\prod_{k \neq i}\left(a_{i}-a_{k}\right)}
$$


We have:

$$
\left.\left(J^{-1} P J^{-1}\right)^{t}\right)_{i n}=\frac{(-1)^{N}}{\prod_{m \neq i}\left(a_{i}-a_{m}\right) \prod_{p \neq n}\left(a_{n}-a_{p}\right)}\left(P_{i n}^{(1)}-P_{i n}^{(2)}\right)
$$

with:

$$
\begin{aligned}
& P_{i n}^{(1)}=\sum_{r=0}^{N-1} \sum_{l=r+1}^{N}(-1)^{l} s_{N-l}\left(a_{1}, \ldots, a_{N}\right) \sum_{k=r+1}^{l} a_{i}^{r+l-k} a_{n}^{k-1}\left[B_{r}, \cdot\right] \\
& P_{i n}^{(2)}=\sum_{r=1}^{N} \sum_{l=0}^{r-1}(-1)^{l} s_{N-l}\left(a_{1}, \ldots, a_{N}\right) \sum_{k=l+1}^{r} a_{i}^{r+l-k} a_{n}^{k-1}\left[B_{r}, \cdot\right] .
\end{aligned}
$$

Subtracting (A.5) and (A.6), by using induction and the identities

$$
\begin{aligned}
& s_{i}\left(a_{1}, \ldots, a_{N+1}\right)=s_{i}\left(a_{1}, \ldots, a_{N}\right)+a_{N+1} s_{i-1}\left(a_{1}, \ldots, a_{N}\right) \\
& s_{i}\left(a_{1}, \ldots, a_{N}\right)=0 \text { if } i<0 \text { or } i>N \\
& \sum_{l=0}^{N}(-1)^{l} s_{N-l}\left(a_{1}, \ldots, a_{N}\right) x^{l}=(-1)^{N} \prod_{i=1}^{N}\left(x-a_{i}\right)
\end{aligned}
$$

one proves that the coefficient of $B_{r}$ in formula (A.4) vanishes if $i \neq n$.

Now let us consider the diagonal terms.

$$
P_{i i}^{(1)}-P_{i i}^{(2)}=\sum_{r=0}^{N}\left(a_{i}^{r} \sum_{l=0}^{N}(-1)^{l} s_{N-l}\left(a_{1}, \ldots, a_{N}\right)(l-r) a_{i}^{l-1}\left[B_{r}, \cdot\right]\right) .
$$

Since

$\sum_{l=0}^{N}(-1)^{l} s_{N-l}\left(a_{1}, \ldots, a_{N}\right) l a_{i}^{l-1}=\left.(-1)^{N} \frac{d}{d x}\left(\prod_{j}\left(x-a_{j}\right)\right)\right|_{x=a_{i}}=(-1)^{N} \prod_{j \neq i}\left(a_{i}-a_{j}\right)$

we get (using (2.8) and (A.2) ) :

$$
P_{i i}^{(1)}-P_{i i}^{(2)}=(-1)^{N} \prod_{j \neq i}\left(a_{i}-a_{j}\right) \sum_{r=0}^{N} a_{i}^{r}\left[B_{r}, \cdot\right]=(-1)^{N}\left(\prod_{j \neq i}\left(a_{i}-a_{j}\right)\right)^{2}\left[A_{i}, \cdot\right]
$$

whence the assertion. 


\section{Appendix B}

Lemma 4 The Poisson tensors $Q$ (2.12) and $R$ (3.2) are not compatible for $N \geq 3$ for any choice of the parameters $a_{1}, \ldots, a_{N}$.

Proof: An explicit computation shows that the Poisson tensor $Q$ can be written in the "coordinates" $\left\{A_{1}, \ldots, A_{N}\right\}$ as:

$$
\{F, G\}_{Q}=\sum_{i, j, k} q_{i j k} \operatorname{Tr}\left(A_{k}\left[\frac{\partial G}{\partial A_{j}}, \frac{\partial F}{\partial A_{i}}\right]\right)
$$

with:

$$
\begin{aligned}
& q_{i j k}=(-1)^{N}\left\{\delta_{i j}\left(\xi_{j} \delta_{j k}+\beta_{j} \frac{\left(1-\delta_{j k}\right)}{\eta_{j k}}\right)+\frac{\left(\beta_{i} \delta_{j k}-\beta_{j} \delta_{i k}\right)}{\eta_{j i}}\right\} \\
& \eta_{i j}=\left\{\begin{array}{ccc}
a_{i}-a_{j} \quad \text { if } i \neq j & \beta_{i}=\prod_{k \neq i} \frac{a_{k}}{\eta_{i k}} \quad \xi_{i}=\sum_{j \neq i} \frac{\beta_{j}}{\eta_{j i}} .
\end{array}\right.
\end{aligned}
$$

Using this expression is easy to evaluate the Schouten bracket of $Q, R$ on the differentials of the functions

$$
F=\operatorname{Tr}\left(A_{1} h\right) \quad G=\operatorname{Tr}\left(A_{2} x\right) \quad H=\operatorname{Tr}\left(A_{2} h\right)
$$

where with $x$ and $h$ we denoted two constant matrices satisfying $[h, x]=x$. We have:

$$
\begin{aligned}
& {[Q, R]_{S}(d F, d G, d H)=} \\
& =(-1)^{N}\left[\xi_{2}-\xi_{1}+\frac{\left(\beta_{1}-\beta_{2}\right)}{\eta_{21}}+\beta_{2} \sum_{j=3}^{N} \frac{1}{\eta_{2 j}}\right] \operatorname{tr}\left(A_{1} x\right)+\beta_{1} \sum_{k=3}^{N} \frac{1}{\eta_{k 1}} \operatorname{tr}\left(A_{k} x\right)(
\end{aligned}
$$

A necessary condition for (B.1) to vanish is that $\beta_{1}=0$, i.e., one of the constants $a_{2}, \ldots, a_{N}$ must be equal to zero. Let us suppose $a_{k}=0, k>2$; then

$$
[Q, R]_{S}(d F, d G, d H)=(-1)^{N}\left(\frac{1}{a_{2}}-\frac{1}{a_{1}}\right) \operatorname{Tr}\left(A_{1} x\right) \neq 0
$$

since $a_{1} \neq a_{2}$.

In the case $a_{2}=0$, we have instead:

$$
[Q, R]_{S}(d F, d G, d H)=(-1)^{N} \operatorname{Tr}\left(A_{1} x\right) \sum_{j=3}^{N} \frac{1}{a_{j}}
$$


If $N=3$ then (B.2) is different from zero, so we can assume $N>3$. But if $a_{2}=0$ and $N>3$ we can consider

$$
F^{\prime}=\operatorname{Tr}\left(A_{1} h\right) \quad G^{\prime}=\operatorname{Tr}\left(A_{2} x\right) \quad H^{\prime}=\operatorname{Tr}\left(A_{3} h\right)
$$

Since

$$
[Q, R]_{S}\left(d F^{\prime}, d G^{\prime}, d H^{\prime}\right)=(-1)^{N+1} \frac{1}{a_{3}} \operatorname{tr}\left(A_{1} x\right) \neq 0
$$

the proof is concluded.

\section{References}

[1] M. Adams, J, Harnad, J. Hurtubise, Darboux Coordinates and LiouvilleArnold Integration in Loop Algebras, Comm. Math. Phys. 155 (385-413), (1993)

M. Adams, J, Harnad, J. Hurtubise, Darboux Coordinates on Coadjoint orbits of Lie Algebras, Lett. Math. Phys. 40 (41-57), (1996).

[2] M. Błaszak, On separability of bi-Hamiltonian chain with degenerated Poisson structures. J. Math. Phys. 39, 3213-3235, (1998)

[3] A. Ballesteros, O. Ragnisco, Classical Hamiltonian systems with sl(2) coalgebra symmetry and their integrable deformations, J. Math. Phys. 43, 954-969, (2002)

[4] A. Ballesteros, M. Corsetti, O. Ragnisco, N-dimensional classical integrable systems from Hopf Algebras, Czech. J.Phys. 46, 1153-1165 (1996).

[5] Yu.A. Drozd, S.A. Ovsienko, V.M. Futorny, On Gel' fand-Zetlin modules, Proceedings of the Winter School on Geometry and Physics (Srni, 1990), Rend. Circ. Mat. Palermo (2) Suppl. No. 26, 143-147, (1991).

[6] Dubrovin B.A. Matrix finite-zone operators, J. Sov. Math. 28, 20-50 (1985).

[7] G. Falqui, F. Magri, M. Pedroni, Bihamiltonian geometry and separation of variables for Toda lattices, J Nonlinear Math. Phys. 8 Suppl., 118-127 (2001).

[8] G. Falqui, M. Pedroni, On a Poisson Reduction for Gel'fand-Zakharevich Manifolds, (nlin.SI/0204050), Rep. of Math. Phys., 50, 395-407 (2002).

[9] G. Falqui, M. Pedroni, Separation of variables for bi-Hamiltonian systems, nlin-si/0204050, to appear in Math. Phys. Anal. Geom (2003). 
[10] G. Falqui, F. Musso, Bi-Hamiltonian Geometries of Gaudin Models: a Case Study, Proceedings of the International Conference SPT 2002 Symmetry and Perturbation Theory, World Scientific, Singapore, 42-50 (2002).

[11] H. Flaschka, J. Millson, The moduli space of weighted configurations on projective space, math.SG/0108191.

[12] M. Gaudin, Diagonalisation d' une classe d' hamiltoniens de spin, J. de Physique 37, 1087-1098 (1976).

See, also, M. Gaudin, La Fonction d' Onde de Bethe, Masson, Paris (1983).

[13] M.I. Gekhtman, Separation of variables in the classical SL(N) magnetic chain, Comm. Math. Phys. 167 (3), 593-605, (1995).

[14] I. M. Gel'fand, I. Zakharevich, On the local geometry of a bi-Hamiltonian structure. In: The Gel'fand Mathematical Seminars 1990-1992 (L. Corwin et al., eds.), Birkhäuser, Boston, 51-112 (1993).

I. M. Gel'fand, I. Zakharevich, Webs, Lenard schemes, and the local geometry of bihamiltonian Toda and Lax structures Selecta Mathematica, new series, 6 131-183 (2000).

[15] J. Harnad, O. Yermolaeva, Superintegrability, Lax matrices and separation of variables, nlin.SI/0303009.

[16] B. Jurčo, Classical Yang-Baxter equations and quantum integrable systems, J. Math. Phys. 30, 1289-1293, (1989)

[17] M. Kapovich, J. Millson, The symplectic geometry of polygons in Euclidean space,J. Differ. Geom. 44, 479-513 (1996)

[18] V. Karimipour, Integrable Structure of the New Calogero Models, J. Math. Phys. 39, 913-920, (1998).

[19] F. Magri, C. Morosi, Quaderno S 19/1984 del Dip. di Matematica dell'Università di Milano, (1984).

[20] F. Magri, Geometry and Soliton Equations, Atti. Acc. Sci. Torino, 124 (Suppl.), 181-209 (1990).

[21] C. Morosi, G Tondo, Quasi-Bi-Hamiltonian systems and separability. J. Phys. A: Math. Gen. 30 (1997), 2799-2806.

[22] M. Pedroni, P. Vanhaecke, A Lie algebraic generalization of the Mumford system, its symmetries and its multi-Hamiltonian structure, Regul. Chaotic Dyn. 3, 132-160, (1998). 
[23] A.G. Reyman, M.A. Semenov-Tian-Shansky, Group-Theoretical Methods in the Theory of Finite-Dimensional Integrable Systems in Dynamical Systems VII, Springer (1994).

[24] D.R.D. Scott, Classical functional Bethe ansatz for $\mathrm{SL}(N)$ : separation of variables for the magnetic chain, J. Math. Phys. 35, 5831-5843, (1994)

[25] E.K. Sklyanin Separation of Variables in the Classical Integrable SL(3) Magnetic Chain, Comm. Math. Phys. 150, 181-192 (1992)

[26] E. K. Sklyanin, Separation of Variables in the Gaudin Model, J. Sov. Math. 47:2, 2473-2488 (1989).

[27] E. K. Sklyanin, Separation of Variables: new trends Progr. Theor. Phys. Suppl. 118 35-60 (1995). 